Cahiers de recherches médiévales

\title{
Brunetto Latini, le Livre dou Tresor et l'histoire sainte
}

Une réception du De ortu et obitu patrum d'Isidore de Séville

\section{Bernard Ribémont}

\section{OpenEdition \\ Journals}

\section{Édition électronique}

URL : https://journals.openedition.org/crm/10772

DOI : $10.4000 / \mathrm{crm} .10772$

ISSN : $1955-2424$

\section{Éditeur}

Honoré Champion

\section{Édition imprimée}

Date de publication : 10 décembre 2008

Pagination : 135-158

ISSN : 1272-9752

Référence électronique

Bernard Ribémont, «Brunetto Latini, le Livre dou Tresor et l'histoire sainte », Cahiers de recherches médiévales [En ligne], 16 | 2008, mis en ligne le 15 décembre 2011, consulté le 15 décembre 2022. URL : http://journals.openedition.org/crm/10772 ; DOI : https://doi.org/10.4000/crm.10772 


\title{
rM
}

\section{Brunetto Latini, le Livre dou Tresor et l'histoire sainte : Une réception du De ortu et obitu patrum d'Isidore de Séville'}

\begin{abstract}
This paper focuses on how Brunetto Latini incorporates into his Livre dou Tresor a section devoted to sacred history. The underlying reason is that Brunetto's conception of history is directed by the notion of law. He is thus led to draw from written life stories of Old Testament prophets and of the disciples of Christ. Foremost among them is De ortu et obitu patrum, written at the end of the VIt century by Isidore of Seville. This study is intended to shed light on the relationship between these two texts.

Résumé : L'objet de ce travail est de se pencher sur la façon dont Brunetto Latini intègre dans son Livre dou Tresor une partie consacrée à l'histoire sainte, démarche motivée par une conception de l'histoire orientée par l'idée de loi. Ceci a conduit le Florentin a utiliser des sources relatant la vie anecdotique de prophètes de l'Ancien Testament, puis de disciples du Christ. Parmi celles-ci, le De ortu et obitu patrum occupe une place essentielle; on tentera ici de mettre en lumière les rapports entre les deux textes.
\end{abstract}

\section{L'encyclopédie, le Miroir du prince, l'histoire et la loi}

Le Livre dou Tresor est composé de trois parties, dont la première peut véritablement être considérée comme une encyclopédie à part entière, dans l'esprit de la famille des grandes encyclopédies du XIII ${ }^{\mathrm{e}}$ siècle $^{2}$. Diverses matières y sont exposées, l'information donnée reposant, comme dans toute encyclopédie médiévale, sur le principe de la compilation, surtout des autorités, et de la recomposition. Ce procédé est explicitement revendiqué par l'auteur, qui use de figures topiques renvoyant à l'acte de compilation, comme le trésor et le butinage des abeilles :

Et si ne di je pas que li livres soit estraiz de mon propre sens ne de ma menue science ; mes il iert aussi come une bresche de mel coillie de diverses flors, car cest

\footnotetext{
${ }^{1}$ Cet article reprend et prolonge une enquête ayant fait l'objet d'un article précédent, «Brunetto Latini, encyclopédiste et traducteur d'Isidore de Séville. L'ordo et l''idéologie': introduction à la matière historique dans le Livre dou Tresor », Lo scaffale della biblioteca scientifica in volgare (secoli XIII-XVI), a cura di Rita Librandi e Rosa Piro, Micrologus lib. 16, Firenze, Sismel, 2006, p. 63-79.

${ }^{2}$ Sur cette notion de famille historique des encyclopédies, voir B. Ribémont, Les Origines des encyclopédies médiévales. D’Isidore de Séville aux Carolingiens, Paris, Champion, Bibliothèque du Moyen Âge, 2001 ; "Around the definition of an encyclopedic genre in the Middle Ages », Premodern encyclopaedic texts, ed. P. Binkley, Brill, Leiden/New York/Köln, 1997, p. 47-61.
}

Cahiers de Recherches Médiévales, 16, 2008 
livres iert conpilez seulement de merveillous diz des actors qui devant nostre tens ont traitié de philosophye, chascun selonc ce que il en savoit partie ${ }^{3}$.

Le livre I du Tresor est donc le produit de cette compilation, touchant à divers domaines: ces derniers ressortissent à deux mouvements qui animent l'encyclopédisme du XIII ${ }^{\mathrm{e}}$ siècle et qui génèrent le plus souvent des ouvrages distincts. Le premier participe de l'encyclopédie 'naturaliste' et touche au quadrivium et aux sciences de la nature; si Brunetto ne s'intéresse pas aux plantes, il consacre en revanche une large partie de ce livre I aux animaux et, également, à la cosmologie, l'astronomie, le comput, la météorologie, la géographie, etc. On y ajoutera un certain intérêt, bien que mesuré, porté aux 'arts mécaniques', ces derniers liés à la ruralité, Brunetto compilant largement Palladius. En second lieu, l'auteur du Tresor inscrit dans son encyclopédie une compilation tenant de l'histoire universelle, illustrée de façon très ample au XIII ${ }^{\mathrm{e}}$ siècle par le Speculum historiale de Vincent de Beauvais, lui-même auteur de la vaste compilation naturaliste qu'est le Speculum naturale. L'œuvre de Vincent illustre bien cette bipolarité entre disciplinae et artes d'une part, historia de l'autre. Or Isidore avait proposé avec les Étymologies une forme d'encyclopédisme global, structuré par la méthode étymologisante, puisque histoire, arts libéraux, sciences naturelles et arts mécaniques sont représentés dans cette œuvre.

Le livre I du Tresor peut être considéré comme préparatoire à l'enseignement du prince, dont l'essentiel repose sur rhétorique, éthique et politique (livres II et III). Cette propédeutique encyclopédique constituerait alors le minimum de culture générale que Brunetto attend d'un gouvernant. Ceci explique probablement la présence assez importante de chapitres historiques dans ce livre I, car l'histoire est sans aucun doute essentielle dans la formation du prince, ce pour deux raisons; l'histoire biblique offre des modèles de comportement, des rois exemplaires et la démarche d'un peuple tel qu'un prince doit le diriger. Du point de vue profane, l'histoire expose l'ensemble des cas qu'un roi est amené à rencontrer: ici encore l'exemple est essentiel, c'est pourquoi l'histoire latinienne se dit largement comme une succession de cas particuliers.

En dépit de cette logique didactique, l'histoire, en tant que discipline, pose un problème à Brunetto, que l'on mesure bien dans la façon dont elle est traitée au regard de la classification des sciences que le Florentin prétend exposer en préambule comme squelette de son ouvrage. L'histoire apparaît au tout début, juste après l'annonce de l'intention encyclopédique, en expliquant que «la premiere partie de cest tresor est autresi come deniers contans, por despendre touzjor en choses besoignables; c'est a dire que ele trate dou comencement dou siecle et de l'ancieneté des vielles estoires, et de l'establissement dou monde $»^{4}$. Brunetto ajoute ensuite qu'elle ressortit à la théorique.

Or la philosophie théorique est subdivisée, comme chez Boèce puis plus tard chez Hugues de Saint-Victor, en trois parties: la théologie, la physique et la mathématique. La mathématique correspond aux arts du quadrivium, la physique

\footnotetext{
${ }^{3}$ Brunetto Latini. Tresor, a cura di P. G. Beltrami, P. Squillacioti, P. Torri, S. Vatteroni, Torino, Einaudi, 2007, p. 6.

${ }^{4}$ Éd. cit., p. 4.
} 
concerne l'étude des « corporaus choses », c'est-à-dire des res du monde sublunaire, « des homes et de bestes, des oiseaus, des poissons, des plantes et des pierres et des autres corporels choses qui sont entre nos $\|^{5}$. Il est donc clair que la référence du prologue aux «vieilles histoires» ne peut concerner que la théologie. Mais, au chapitre 3, Brunetto définit bien la théologie comme la science des substances séparées : «... teologie, qui trespasse le ciel et nos mostre les natures des choses qui n'ont point de cors ne ne conversent entre les corporaus choses » (p. 8).

L'histoire revient, 'par la marge', dans le chapitre 4 consacré à la philosophie pratique. Cette inscription passe par le biais de la rhétorique et d'une référence à Cicéron; Brunetto relie une conception morale et utilitaire de l'art de la parole à la prédication et à l'édification, ce qui lui permet de faire référence à l'Écriture :

(...) c'est la science qui adreça le monde premierement a bien fere, et qui encor l'adresce por la predicacion des sainz homes, per les divines Escritures et per la loy que les genz governe a droit et a justise. (p. 12)

Ce renvoi à la Bible est en relation avec la loi et la justice, ce qui concerne aussi l'éthique et la politique. Si la dimension historique n'est pas explicite, elle est de fait introduite par tramage si l'on considère que, premièrement, la Bible est l'histoire d'un peuple - et c'est bien ainsi que Brunetto va l'utiliser - et que, deuxièmement, l'explication de la Bible passe par une exégèse dont le premier stade est l'historia. Pour bien comprendre une telle logique, on peut mettre le Tresor en parallèle avec le Didascalicon de Hugues de Saint-Victor ${ }^{6}$, dont le dernier livre (6) est consacré à l'Écriture sainte. Dans le premier chapitre de ce dernier livre, le Victorin rappelle que l'ordre de la lecture se présente selon quatre aspects : selon les disciplines, les livres, la narration et l'exposition, et qu'il lui faut appliquer cela à l'Écriture (ordinem legendi supra quadrifarium esse commemoravi, alium in disciplinis, alium in libris, alium in narratione atque alium in expositione). Ce qu'il entend alors par l'ordre dans les disciplines (De ordine qui est in disciplinis) concerne les trois sens courants selon l'exégèse. Le premier est donc l'historia auquel Hugues consacre son chapitre 3. Celle-ci est considérée comme propédeutique à l'allégorie (neque ego te perfecte subtilem posse fieri puto in allegoria, nisi prius fundatus fueris in historia) et Hugues se livre à une défense en règle de l'histoire: il ne faut pas négliger les détails, même s'ils paraissent inutiles. L'histoire représente les fondations de la lecture sacrée, elle permet d'admirer les réalisations de Dieu (Habes in historia quo Dei facta mireris) ${ }^{7}$. Après cette démonstration, Hugues indique de fait ce que doit être le début d'un regard portant sur l'histoire universelle, en faisant un résumé, sous forme de courtes phrases, des

\footnotetext{
${ }^{5}$ Éd. cit., p. 10. On remarquera que Brunetto énonce ces res naturales dans un ordre hiérarchique descendant.

${ }^{6}$ Pour le texte latin, utiliser l'édition de Ch. Buttimer, Washington 1939. Texte disponible sur internet http://freespace.virgin.net/angus.graham/Hugh.htm ; il existe une bonne traduction de M. Lemoine, Paris Cerf (Sagesse chrétienne), 1991.

${ }^{7}$ Sur la conscience de l'histoire de Hugues de Saint-Victor, on pourra lire les belles pages de M.-D. Chenu, dans La Théologie au XII siècle, Paris 1966, p. 64ss.
} 
épisodes majeurs marquant la chronologie de l'Ancien Testament, de la Création jusqu'à la naissance de Jésus.

C'est un tel programme que Brunetto va suivre. Après en avoir terminé avec le détail concernant la philosophie et ses parties, l'encyclopédiste florentin aborde, comme il l'avait annoncé au prologue, la philosophie théorique par les «vieilles histoires» et donc par l'origine, "Coment Dieu fist toutes choses au comencement», comme l'annonce la rubrique du manuscrit de l'Escorial. Cela permet à Brunetto une organisation des chapitres suivants dans un ordre descendant, tel qu'on le trouve aussi dans le De proprietatibus rerum de Barthélemy l'Anglais : Dieu, anges, homme. L'homme possède mémoire et raison, mais il a été trompé par «les mauvais anges», ce pourquoi il a fallu établir la loi sur la terre. La loi se décompose en loi divine et humaine. Il existe alors, à partir de Moïse, une véritable chronologie de la loi, une permanente translatio legis que Brunetto expose en fin de chapitre 17 et qui, de fait, ouvre, en toute logique, la voie à la partie historique du Tresor.

Il y a donc une erreur d'appréciation à considérer le Livre dou Tresor dans son ensemble comme une encyclopédie structurée, ainsi que l'annonce Brunetto dès le début, par une classification des sciences de type stoïcien. Car, effectivement, le livre I échappe à cette classification, pour deux raisons principales : parce qu'il est une encyclopédie 'naturaliste' et parce qu'il contient une synthèse d'histoire universelle. Dans ces deux directions, le texte se doit de proposer un ensemble d'informations sur choses et événements qui ne peuvent directement s'apparenter à la rhétorique, l'éthique ou la politique. C'est la raison pour laquelle, si les autorités premières sont Cicéron et Aristote, Isidore de Séville représente, au moins indirectement, l'exemple pour le livre I d'une encyclopédie globale, intégrant l'ensemble des données de cette 'culture générale' nécessaire au litteratus rex, dans laquelle l'histoire joue un rôle particulier, car elle est utile en elle-même comme exemple, comme l'avait bien remarqué Vincent de Beauvais :

Porro quarta et ultima videlicet hystorialis licet ad philosophiam directe non pertineat, eo quod singularia rerum gesta tantum enarrat, de quibus scilicet singularibus secundum Aristotilem ars non est, plurimum tamen et admirationis et recreationis et utilitatis habet, ut iam superius dictum est $(1,16)^{8}$

On trouve une idée proche chez Isidore qui définit l'histoire comme la narratio rei gestae: elle est liée à la grammaire (Haec disciplina ad grammaticam pertinet) parce qu'elle met en écrit ce qui est digne de mémoire (quia quidquid dignum memoria est litteris mandatur). C'est bien de cette mémoire dont le prince a besoin.

\section{Isidore de Séville}

Bien que jamais cité dans le livre I du Tresor, Isidore de Séville fait donc partie de ces 'philosophes' dont Brunetto se réclame et auprès de qui il s'alimente. Or, lorsqu'il est question de l'évêque de Séville en matière d'encyclopédie, c'est aux

${ }^{8}$ Le texte est disponible, dans l'édition de Douai citée ici, sur le site internet de l'Atelier Vincent de Beauvais http://atilf.atilf.fr/bichard/ 
Étymologies que l'on songe en premier lieu. Ce texte en effet peut être considéré comme fondateur de l'encyclopédisme médiéval et, encore au XIII $I^{\mathrm{e}}$ siècle, à une époque où le savoir savant a considérablement évolué et progressé par rapport au contenu de l'encyclopédie d'Isidore, celle-ci demeure au rang des sources les plus citées. Un ouvrage comme le De proprietatibus rerum par exemple est empli de $u t$ dicit Ysidorus. Si les encyclopédistes du XIII ${ }^{\mathrm{e}}$ siècle puisent largement dans des sources arabo-latines, Isidore fait toujours figure d'autorité et il est généralement utilisé de trois façons, le plus souvent avec une référence explicite - témoignage du rôle d'autorité que l'évêque de Séville continue d'avoir au XIII ${ }^{\mathrm{e}}$ siècle :

1) au 'degré zéro', en tant que simple ut dicit, souvent associé à une autre autorité, lorsque l'auteur expose un donné qui se trouve aussi dans les Étymologies. À propos de la coriandre par exemple, au livre XVII du De proprietatibus rerum, Barthélemy l'Anglais met Papias et Isidore côte à côte : Canibus autem hec herba est cum semine venenosa. Nam interficit eos, si ab eis aliquotiens assumatur, ut dicit Isidorus et Papias.

2) L'encyclopédiste peut se saisir d'un contenu objectif et naturaliste des Étymologies : description, propriété d'un élément exposé. Pour rester sur l'exemple du livre sur les plantes de Barthélemy l'Anglais, celui de la coloquinte: Hec herba quasi vitis se diffundit in sepibus, fructu modico et rotundo, ut dicit Isidorus libro $17^{10}$.

3) L'usage le plus attendu, et le plus fréquent, est évidemment la référence étymologique. L'étymologisme isidorien ne fait plus guère partie, au XIII siècle, des méthodes opératoires d'investigation. Cependant, l'encyclopédie conserve les traces de l'autorité de l'étymologie et surtout utilise celle-ci comme système commode de présentation, d'introduction. Il est ainsi fréquent qu'une rubrique commence par une référence étymologique permettant en quelque sorte de mettre en avant l'objet décrit: Cipressus grece dicitur ciparissus, ut dicit Isidorus libro 17, quia caput eius a rotunditate in acumen erigitur, exemple-type d'introduction, comme ici le cyprès du livre XVII du De proprietatibus rerum ${ }^{11}$.

Brunetto Latini procède différemment de Barthélemy l'Anglais ou de Vincent de Beauvais quant à la référence isidorienne; on constate en premier lieu qu'Isidore n'est jamais explicitement cité au livre I. D'autre part, la méthode étymologique est complexe à suivre dans la mesure où Brunetto a choisi d'écrire en vernaculaire $^{12}$, ce qui explique l'absence de présentation étymologisante chez Brunetto. C'est donc plutôt des éléments de contenu qui intéressent le Florentin et son attention va davantage se tourner, en matière d'histoire naturelle, vers des auteurs plus complets qu'Isidore, en particulier vers les anciens qui ont servi de

\footnotetext{
${ }^{9}$ Éd. I. Ventura, Turnhout, Brepols, 2007, p. 71.

${ }^{10} \mathrm{Ibid}$.

${ }^{11}$ Ibid., p. 55.

${ }^{12}$ La traduction des étymologies pose toujours problème; on le voit par exemple chez Jean Corbechon, traducteur de Barthélemy l'Anglais; voir mon article, «Jean Corbechon, traducteur encyclopédiste au XIV ${ }^{\mathrm{e}}$ siècle », Cahiers de Recherches Médiévales, 6, 1999, p. $75-98$.
} 
source à l'évêque de Séville. On peut le mesurer aux emprunts très lourds faits à Solin.

Il en va de façon un peu différente pour ce qui concerne l'histoire sainte pour laquelle, à côté des textes sacrés eux-mêmes, les textes isidoriens, Sentences, Étymologies et De ortu et obitu patrum fournissent à Brunetto un réseau d'informations résumées utiles à son exposé. La démarche latinienne obéit en la matière à une logique claire : tout d'abord, l'histoire à écrire est une conséquence de la loi, nécessaire à l'humanité, d'abord dictée par Dieu, le premier créateur de la loi. Après Dieu, c'est le prince qui est le garant de l'ordre sur terre (le pax et ordo qui régit la conception augustinienne du pouvoir). C'est sans doute là l'élément le plus important et qui s'accroche au mieux à la problématique du Tresor.

Il est ainsi très significatif que Brunetto, dans le chapitre 17, «Coment loi fu premierement establie », présente celle-ci, non en contenu, non dans une perspective juridique, mais bien dans un sens historique, comme progression attachée exclusivement, à partir de Dieu, à un puissant. Pour ce faire, Brunetto reprend le livre 5 des Étymologies d'Isidore, qu'il traduit presque littéralement, selon un procédé de 'compilation orientée'13 : il s'agit ici pour le Florentin d'insister sur le caractère fondateur d'une histoire partant du Dieu de l'Ancien Testament pour arriver à l'ère chrétienne, porteuse des valeurs que le prince se doit de promouvoir et de défendre. La succession des chapitres obéit à cette logique. Ainsi, le chapitre 18 «Ci dit de la divine loy», dans son début compilé des Sentences $(1,20)^{14}$, s'inscrit dans la continuité de l'exposé sur la définition de la loi divine: la loi divine est naturelle ( «La divine loi est por nature»), parce qu'elle émane de Dieu. L'encyclopédiste ne reprend donc pas les propos d'Étymologies 5, 4 (Ius naturale [est] commune omnium nationum), pour insister sur l'ancrage divin de la loi, révélé aux hommes par l'Ancien Testament. Le passage vers le Nouveau se fait par le truchement de la hiérarchie céleste: lorsque l'on franchit le temps séparant le Nouveau Testament de l'Ancien, les hommes ont progressé, grâce à la loi ; les anges ne les méprisent plus :

Por ce avenoit il au viel tens que, quant aucuns hom saluoit les angles, il ne li rendoi[en]t salus, ainz li despisoient ; mes ou Nuef Testament lisons nos que Gabriel salua Marie. Et quant Jahanz salua l'angle, il respondi en tel maniere : «garde, fist il, ne fere, car ge sui ton serf et [de] t[es] frere[s]. (p. $34-$ je modifie la ponctuation))

Ce progrès est cependant ambigu : Brunetto, en effet, remarque que, dans les temps anciens, les péchés étaient véniels, par ignorance de la nouvelle loi. Celle-ci révélée aux hommes, ceux-ci n'en sont que plus étroitement justiciables, car ils ont le loisir d'exercer leur libre arbitre en toute connaissance de cause. C'est pourquoi la justice des princes devient une nécessité :

\footnotetext{
${ }^{13}$ Voir, pour une analyse du passage, mon art. cit., "Brunetto Latini, encyclopédiste et traducteur d'Isidore de Séville. L'ordo et l''idéologie' : introduction à la matière historique dans le Livre dou Tresor ».

${ }^{14}$ Éd. P. Cazier, CCSL 111, p. 71-2.
} 
mes por ce que comander ou establir loi poi vaut entre los homes se il ne fust aucuns qui les peust contraindre a garder la loi, convint il que, por esausier justice et por mortefier le torfaiz, fussent establis rois et seignors de maintes manieres. (p. 34-36)

Le chapitre 19, «Coment rois et roiaumes furent premierement» conclut cette introduction/justification concernant l'histoire et, en démarquant Étymologies, 5,38 , Brunetto achève de présenter sa chaîne logique : la loi naturelle vient de Dieu, elle est révélée aux premiers hommes par les prophètes, puis renouvelée par la parole de Jésus, propagée par les apôtres auprès d'une humanité dont l'ordre doit être assuré par la justice des princes. Politique et justice s'enchaînent dans l'histoire de la longue durée qui, depuis la Création, s'égrène dans les six âges de l'homme rappelés en fin de chapitre. Le prince, en premier lieu, est celui qui se doit de connaître cette histoire : ce n'est donc pas un hasard si le premier chapitre (19) de la progression historique s'intitule ainsi. D'autre part, en toute logique, l'histoire contée au prince est avant tout exemplaire. Or, les meilleurs exemples pour les gouvernants sont à trouver auprès des figures illustres que les textes d'autorité ont pu livrer; il y a aussi du De viris illustribus dans la démarche de Brunetto ${ }^{15}$. Tous ces principes posés, il reste à Brunetto à dérouler le temps selon son découpage le plus traditionnel pour, dans le cadre du sixième âge, arriver à la période contemporaine, celle qui s'ancrera dans les événements et permettra à Brunetto de faire aussi valoir ses opinions guelfes.

\section{Brunetto, lecteur $d u$ De ortu et obitu patrum}

Mais avant de dérouler l'histoire contemporaine, c'est l'histoire des figures bibliques qui importe au Florentin; pour faire un exposé, qu'il veut condensé, des exempla que ces figures représentent, Brunetto dispose bien entendu de la Bible et de la Glose, mais il va en fait se tourner largement vers un ouvrage d'Isidore qui, en quelque sorte, lui facilite la tâche, parce que lui proposant une histoire déjà résumée : il s'agit du De ortu et obitu patrum (Doop) qu'il va utiliser dans la partie du Tresor qui est consacrée aux prophètes et aux apôtres (chap. XLIV-LXXXIV). Ces quarante et un chapitres sont très courts, certains comportant seulement quatre lignes dans l'édition Beltrami : on sent chez Brunetto le désir de fournir surtout une liste, un ensemble de repères onomastiques: la vie du saint ou du prophète est très rapidement esquissée, sur le modèle du martyrologe, qui est au demeurant celui de la source isidorienne de Brunetto.

La première question qui se pose est celle du choix et de l'utilisation des sources; le Doop comporte en effet, dans sa version la plus complète, 85 rubriques et Brunetto n'en utilise qu'une petite moitié. Il paraît donc légitime de s'interroger sur la démarche du Florentin, sur la sélection qu'il opère. Mais, en ce domaine, il est très délicat d'émettre de fermes hypothèses, car d'une part la tradition manuscrite du Doop n'est pas linéaire et, d'autre part, il apparaît comme quasiment impossible de

${ }^{15}$ On notera que le De ortu et obitu patrum a connu une tradition manuscrite, à partir du XII ${ }^{\mathrm{e}}$ siècle, l'associant aux De Viris illustribus d'Isidore, de Gennade, de Jérôme et d'Ildefonse de Tolède ; voir l'édition du Doop par C. Chaparro Gómez, Paris, Belles Lettres, 1985, p. 10, 93 (toutes les références se feront à cette édition, que nous appellerons aussi «version commune »). 
savoir sur quels manuscrits a travaillé Brunetto. On peut cependant, sans pouvoir véritablement apurer la question, avancer quelques éléments.

\section{Titres, rubriques et tradition manuscrite}

L'étude de la tradition manuscrite du Doop révèle que huit chapitres concernant des prophètes mineurs ont été rajoutés au texte initial d'Isidore, probablement à partir d'un texte grec traduit en latin, les Vitae prophetarum: il s'agit des chapitres 46 (Micheas), 47 (Naum), 48 (Abacuc), 51 (Zacharias), 54 (Achias), 55 (Iaddo), 56 (Azarias) et 57 (Zacharias). Ces huit chapitres se retrouvent dans deux familles, $\Gamma$ et $\Delta$ : la première famille offre la version courte du Doop, à l'exception de deux manuscrits $\mathrm{H}$ et I, interpolés plus tardivement. La seconde famille contient six manuscrits de la version longue, dont cinq (VDLUf) sont apparentés, sous-famille à laquelle il faut ajouter $\mathrm{Z}^{16}$, qui cependant omet le chapitre 46. À part, le manuscrit $\mathrm{G}$ appartient à la tradition 'pseudo-isidorienne' et comporte les huit chapitres avec en sus, dans la partie réservée aux personnages du Nouveau Testament, diverses interpolations devant beaucoup au Breviarum Apostolorum ${ }^{17}$, comme le chapitre 80 qui présente un résumé de ce qui est dit de la vie des disciples de Jésus dans le Doop.

Or, premièrement, Brunetto inclut dans son Tresor une rubrique sur Achias et une sur Jado, calquées sur celles de la version longue du Doop :

\begin{tabular}{|l|l|}
\hline Doop (54) & \multicolumn{1}{|c|}{ Tresor (I, 52) } \\
\hline & De Achias le prophete \\
Achias Silonites, de ciuitate Heli sacerdotis, & Achias [fu] prophete de la cité Helye. Il dit \\
ubi primum tabernaculum et arca Domini & lonc devant le roi Salemon que il deguerpiroit \\
erat; Salomoni regi praedixit quod per & Dieu et sa loy por une feme. Et quant il morut \\
mulieres gentium alienigenerarum a & Son cors fu enterré joste un chasne en Silo. \\
mandatis Domini declinaret. Mortuus est & \\
ergo Achias et sepultus est iuxta quercum in & \\
Silo. & \\
\hline
\end{tabular}

\begin{tabular}{|c|c|}
\hline Doop (55) & Tresor (53) \\
\hline $\begin{array}{l}\text { Iaddo, in Samaria natus; hic est qui ad } \\
\text { Hieroboam immolantem uitulis missus in } \\
\text { sermone Domini uenit eumque arguit } \\
\text { immolantem. Hunc ad propria reuertentem } \\
\text { leo in uia strangulauit sepultumque tenet } \\
\text { Bethel et prophetam illum qui eum fefellerat. }\end{array}$ & $\begin{array}{l}\text { De Jado le prophete } \\
\text { Jado prophete nasqui en Samaria. Il fu } \\
\text { envoiés [a] Jerob[o]am, qui sacrifioit les veels } \\
\text { a Dieu, que il demorast avec lui ; mes il [n']i } \\
\text { demora, et por ce il avint que quant il s'e[n] } \\
\text { reparoit un lion l'estrangla, por ce qu'il avoit } \\
\text { failli a son compaignon. Puis fu enterré Jado } \\
\text { en Bethleem }\end{array}$ \\
\hline
\end{tabular}

D'autre part, la version longue du Doop contient deux chapitres sur Zacharie. Le premier (57) est exclusif de la famille 'longue', alors que le second (64), de la

\footnotetext{
${ }^{16}$ Voir l'introduction éd. cit., p. 75-90.

${ }^{17}$ Texte du VI $I^{\mathrm{e}}$-début VII ${ }^{\mathrm{e}}$ s. qui établit avec autorité la prédication de Jacques en Espagne.
} 
version commune, est dévolu à Zacharie et Élisabeth, mentionnant laconiquement leur stérilité première transformée en fécondité ${ }^{18}$. Ce dernier chapitre est, dans le Doop, précédé de Tobie, les Trois enfants, Esdras, Zorobabel, Esther, Judith. Dans le Tresor, la suite est la suivante: Tobie, les Trois enfants, Esdras, Zorobabel, Esther, Judith, Zacharie. Elle est donc identique au Doop, à ceci près que Brunetto substitue au chapitre Zacharie/Élisabeth de la version commune, le chapitre Zacharie de la version longue:

\begin{tabular}{|c|c|}
\hline Doop (57) & Tresor (60) \\
\hline $\begin{array}{l}\text { Zacharias, propheta atque sacerdos, filius } \\
\text { Ioiade sacerdotis, qui et Barachias } \\
\text { cognominatus est; quem congregatus in } \\
\text { atriis domus Domini populus, iuxta Ioas } \\
\text { regis Iuda imperium, missis extincxit } \\
\text { lapidibus. Hunc sublatum continuo } \\
\text { sacerdotes iuxta patrem suum sepelierunt in } \\
\text { Hierusalem. }\end{array}$ & $\begin{array}{l}\text { Zacharias vaut autant a dire come memoire de } \\
\text { Damedieu; et fu prophete et provoire, fiz fu } \\
\text { Joiade le preste, qui avoit en sornom } \\
\text { Branchias, qui fu lapidés dou pueple por le } \\
\text { comandement dou roi de Juda encoste l'autel } \\
\text { dou temple. Mes les autres provoires } \\
\text { l'ensevelirent en Jerusalem. }\end{array}$ \\
\hline
\end{tabular}

On pourra remarquer sur les deux exemples précédents que Brunetto ne suit pas exactement le texte du Doop ; j’y reviendrai ${ }^{19}$. Mais auparavant, poursuivons la comparaison des chapitres, qui nous amène aux constats suivants :

1) Si Brunetto reprend deux chapitres de la version longue, en revanche, il n'utilise pas les cinq autres supplémentaires.

2) Le Florentin clôt la partie vétérotestamentaire de son encyclopédie par une rubrique sur les Macchabées. Or, un seul manuscrit (de Vérone) nous est parvenu contenant une rubrique sur ces derniers ${ }^{20}$. Arévalo reproduit un tel chapitre dans son édition $^{21}$, mais son texte ne correspond pas à celui donné par le manuscrit. Arévalo le donne après Zorobabel, Esther, Judith et Brunetto dans la liste Zorobabel, Esther, Judith, Zacharie, Macchabées. Dans le manuscrit de Vérone, la suite est Esther, Judith, Macchabées, Zacharie et Élisabeth. De plus, le texte de Brunetto ne correspond en rien à celui de Vérone. À lire le propos de l'encyclopédiste florentin, on a l'impression qu'il ne compile pas une rubrique quelconque du Doop, mais qu'il rappelle seulement, et de façon particulièrement succincte, la généalogie biblique des Macchabées, renvoyant in fine et fort rapidement son lecteur à la Bible :

De Machabeu et de sa vie

Machabeu vaut tant a dire come noble [et] trionphanz. [Et] furent .v. Machabeus fiz Matatie, ce sont J[ehans], J[ude]s, Ele[az]a[r], [Symons] et Jonatas. Et qui voudra

\footnotetext{
${ }^{18}$ Rappelons qu'il y a de nombreux Zacharie dans la Bible : ici, l'un est fils de Barachie (ou Bèrèkia) - il s'agit du prophète (cf. Zach., 1) ; l'autre est fils du prêtre Jéhojada, mort lapidé, dont l'histoire est contée en 2 Chr., 24, 20-22. La confusion vient sans doute de Mathieu (23, 35), erreur non reproduite par Luc.

${ }^{19}$ Voir infra, Brunetto traducteur et adaptateur.

${ }^{20}$ Vérone, BC LVI (ms. O) ; le chapitre sur les Macchabées est au fol. 34.

${ }^{21}$ Reprise dans $P L 83$.
} 
savoir les victoires que ceaus orent sor les rois de Perse et les granz choses que il firent, si lisse l'estoire qui le conte mot a mot diligenment en la grant Bible.

3) Le manuscrit de Vérone ne contient que la version courte du Doop: Brunetto ne peut donc avoir trouvé dans un tel manuscrit les deux chapitres sur Zacharie et sur Jado.

4) Si l'on compare les chapitres du Tresor et du Doop concernant Élie, on remarque que Brunetto termine sa rubrique par un ajout qui ne figure pas dans la version commune du Doop. Cependant, ce texte, édité par Arévalo, figure dans un manuscrit nous étant parvenu, manuscrit ( $\mathrm{I}-\mathrm{VIII}^{\mathrm{e}} \mathrm{s}$.) considéré comme interpolé, celui de Londres (Harley 5041) :

\begin{tabular}{|l|l|}
\hline Doop $^{22}$ & \multicolumn{1}{|c|}{ Tresor (46) } \\
\hline Elias Thesbites de terra Arabum, de tribu & Cist Hely fu de la lignee Aaron. Et quant il \\
Aaron, cum esset in utero matris suae & vint a sa naissance, Sobi son pere sonja que \\
Galaath, Sobac pater ejus somnium vidit, & homes vestus de blanches robes prenoi[en]t \\
quod nascentem Eliam viri candidis vestibus & Helyas et l'envolopoient en blancs dras, et \\
salutabant, igneisque eum vestimentis & puis li donoient feu a mangier; et quant il \\
obvolvebant, atque pro cibo ignem ei ad & s'esveilla, il enquist a[s] prophetes que ce \\
nutriendum subministrabant. Hoc visum & puet estre, et il distrent: «ne doute pas, car \\
pater ejus prophetis in Jerosolymis indicavit, & ton fis sera lumiere et parlieres de sciences, et \\
hocque ab eis accepit responsum: «Ne & il jugera Israel.» \\
timueris, inquiunt, erit enim natio ejus & \\
lumen, verbumque ejus fixis sententiis. & \\
Judicabit enim Israel in gladio et igne. » & \\
\hline
\end{tabular}

5) Brunetto compile ses chapitres 'par blocs'. Après en avoir terminé avec les six âges, en indiquant qu'il était légitime, parce que la «vieille loy, la ou ele ne fu pas remue » était conservée, de parler des « maistres de cele loy et la vie de chascun en ceste maniere» (p. 78), il commence par David (chap. 44), suivi de Salomon, Hélie, Élisée, Isaïe, Jérémie et Daniel, qui tous se suivent dans le Doop (33-40). Brunetto a donc éliminé les 32 premiers chapitres du texte isidorien, de Adam à Samuel. Il saute ensuite, de la version longue, de Osée à Achias (Aia) (41-54), reprend Jado, saute Azaria et Zacharie (qu'il mettra plus loin, on l'a vu), puis reprend la série Tobias, les trois enfants, Esdras, Zorobabel, Esther, Judith, Zacharie (substitué); vient ensuite le chapitre sur les Macchabées, qui clôt la liste vétérotestamentaire. Brunetto tient à bien marquer la césure - c'est important car il raisonne en termes de loi -et il insère deux chapitres de conclusion/introduction aux titres significatifs de « Des livres dou Viel Testatment» et « Ci commence la novelle loi » respectivement. Dans cette dernière rubrique, il rappelle la généalogie du Christ depuis Abraham et termine avec la mère de Jésus. L'histoire de Marie sert ainsi de support aux deux chapitres suivants qui inaugurent les personnages liés à la Nouvelle loi. Brunetto énumère alors dix-huit personnages étroitement liés à la vie de Jésus : Jean-Baptiste, Jacques d'Alphée, Judas frère de Jacques, Jean, Jacques fils

${ }^{22}$ P.L. 83, c. 141, 64. 
de Zébédée, Pierre, Paul, André, Philippe, Thomas, Barthélemy, Matthieu, Mathias, Simon, Luc, Marc, Barnabé, Timothée, Tite et termine avec un nouveau chapitre général sur le Nouveau Testament. Si l'on compare la série latinienne avec la vulgate du Doop, on constate que Brunetto reprend l'ordre des sept derniers disciples, mais n'offre pas le même pour les onze premiers qui, dans le Doop, apparaissent dans la série : Jean-Baptiste, Marie, Pierre, Paul, André, Jacques fils de Zébédée, Jean, Philippe, Thomas, Barthélemy, Mathieu, Jacques d'Alphée, Judas. Dans cette série cependant, le sous-ensemble Philippe, Thomas, Barthélemy, Mathieu est commun. Enfin, Brunetto ne reprend pas le chapitre 80, venu du Brevarium Apostolorum et que d'ailleurs certains manuscrits du Doop rejettent à la fin.

À partir de ces quelques points concernant l'organisation des rubriques et la tradition manuscrite, l'on peut avancer une première hypothèse sur la façon dont Brunetto a utilisé Isidore. Le Florentin a travaillé sur un ou des manuscrits du Doop contenant une version longue, peut-être incomplète, mais incluant les chapitres 51 (Zacharie), 54 (Achias) et 55 (Jado) qu'il décalque; il a connu des versions interpolées, telle celle figurant dans le manuscrit I. D'autre part, Brunetto a eu très certainement sous les yeux un manuscrit contenant, comme celui de Vérone, le chapitre sur les Macchabées. Il est cependant ici bien difficile de cerner la réalité de la compilation. Le texte que fournit le Tresor n'a rien à voir avec celui de $\mathrm{O}$, ni avec celui que publie Arévalo. Comme on l'a vu plus haut, la rubrique de Brunetto est en fait composée de trois parties: la première donne une étymologie, la deuxième une généralité qui ne saurait renvoyer à aucune source particulière, et la troisième un 'abandon' laissant au lecteur le soin d'aller se renseigner dans la Bible. L'étymologie' est construite par Brunetto lui-même - elle ne figure pas comme telle chez Isidore - et vient du Liber prooemiorum, texte qui précède souvent, dans les manuscrits des œuvres d'Isidore, le Doop. Dans une rubrique consacrée au livre des Macchabées, l'évêque de Séville indique en effet: Praenotant autem praelia inter Hebraeorum duces gentesque Persarum, pugnam quoque Sabbatorum, et nobiles Machabaei triumphos, foedus quoque amicitiarum cum Romanorum ducibus, actaque legationum ${ }^{23}$.

Ajoutons que la place de la rubrique sur les Macchabées, même si celle-ci est proche de celles parlant d'Esther et de Judith, ne correspond pas non plus à l'ordre le plus habituel de la Vulgate. Il paraît donc raisonnable, pour conclure sur ce point, que Brunetto a effectivement travaillé sur un manuscrit qui contenait un chapitre sur les Macchabées ; ou bien ce manuscrit appartenait à une famille qui ne nous est pas parvenue, et Brunetto a décalqué le latin. Ou bien le manuscrit contenait la rubrication, qui a donné l'idée à Brunetto d'inclure un discours de son crutoutefois mâtiné d'Isidore - dans son Tresor. Ou bien encore-pour des raisons matérielles (manuscrit endommagé ?) ou de contenu - le texte ne satisfaisait pas l'encyclopédiste qui a cherché ailleurs son information-, dans le Liber prooemiorum, la Bible et sa propre mémoire. Il me paraît difficile d'aller plus avant à ce sujet.

${ }^{23}$ P.L. 83, c. 175 (c'est moi qui souligne). 


\section{Brunetto traducteur et adaptateur}

Venons-en à présent au contenu du texte de Brunetto, en regard de celui du Doop. Il est clair, à l'examen des différents chapitres du Tresor comparés à ceux du Doop, que Brunetto s'inspire très largement du Doop pour égrener ce qu'il entend exposer des vies des prophètes et apôtres. Mais, comme la plupart des 'traducteurs' médiévaux, Brunetto est avant tout un translator et un adaptateur. Je me propose donc d'examiner de quelle façon il 'translate' le texte d'Isidore de Séville.

\section{RESTER PRES DE SA SOURCE...}

Le 'degré zéro de la translatio consiste à compiler le texte latin et à le traduire/adapter en demeurant au plus près; s'il ne s'agit pas à proprement parler d'une traduction au sens moderne, le procédé en est toutefois assez proche. Pour un certain nombre de chapitres, Brunetto peut effectivement apparaître comme un traducteur; tel est le cas du chapitre sur Jérémie ou de celui sur Zorobabel :

\begin{tabular}{|l|l|}
\hline \multicolumn{3}{|c|}{ Doop (38) } & \multicolumn{1}{|c|}{ Livre dou Tresor (49) } \\
\hline Hieremias, ex tribu sacerdotali ortus in uico & De Jer[e]mie et de sa vie \\
Anathot, qui est tribus milibus ab & en un chastel qui a nom Anatot, a .iii. liues de \\
Hierusalem; sacerdos in Iudaea positus, & Jerusalem; il fu prestes en Judee et fu sacrez \\
propheta in gentibus consecratus; antequam & a prophete. Avant qu'il nasquist fu il coneu, \\
plasmaretur, agnitus; antequam procederet, & et li fu comandé qu'il mantenist virginité. De \\
sanctificatus et, ut uirgo permaneret, & sa enfance comença il a preechier et oster les \\
admonitus. Hic praedicare puer coepit & genz de pechiez, et convertir, les a penitence. \\
arguens populum ob delicta et saepius & Mainz maus [li] furent faiz dou cruel pueple, \\
cohortans ad poenitentiam; sed plebis & car il fu mis en chartre et fu getez en un lac et \\
perfidae saeuientis crudelitate adficitur saepe & fu [cei]nt de cha[enes]. Et en la fin fu lapidez \\
atque ligatur, in carcerem etiam mittitur, & en Egypte; et fu enseveliz la ou li rois \\
proicitur in lacum, ad ultimum apud Tafnas & Pharaon manoit, et son sepulcre e[s]t en grant \\
in Aegyptum a populo lapidatur. Sepultus in & reverance entre les egypciens por ce que il les \\
eo loco ubi Pharao rex Aegypti habitauit. & delivra des serpens. \\
Verumtamen, quia precibus suis, serpentibus & \\
ab eo loco fugatis, Aegyptios a noxio aspidum & \\
morsu eripuit, insigni eum ueneratione & \\
amplissimoque honore idem Aegyptii colunt & \\
sepulcrumque eius insigni cultu hactenus & \\
uenerantur. &
\end{tabular}




\begin{tabular}{|c|c|}
\hline Doop (61) & Livre dou Tresor (57) \\
\hline $\begin{array}{l}\text { Zorobabel et Neemias, ex tribu Iuda, sub } \\
\text { Dario rege templum Domini aedificant, } \\
\text { muros Hierusalem exstruunt atque Israhel ad } \\
\text { pristinum statum reducunt; cultum quoque } \\
\text { religionis iusque sacerdotum uel leuitarum } \\
\text { restituunt; idem et ipsi in Hierusalem sepulti } \\
\text { quiescunt. }\end{array}$ & $\begin{array}{l}\text { De Zorobabel } \\
\text { Zorobabel et N[ee]mias dou lignage Juda } \\
\text { furent provoires et prophetes. Et redifierent le } \\
\text { temple Dieu au tens que Daires li fiz Iastapis } \\
\text { fu rois de Perse. Il firent les murs de } \\
\text { Jerusalem et retornerent Israel en son premier } \\
\text { estat, et estorerent les contenemenz de la } \\
\text { religion et la raison des prevoires. Et furent } \\
\text { ensevelis e[n] Jerusalem. }\end{array}$ \\
\hline
\end{tabular}

On voit sur ces deux exemples que le texte français, bien que très proche du latin, n'y est pas tout à fait fidèle, du moins en rapport à la version commune du Doop; dans le cas de Zorobabel par exemple, le Tresor précise le nom du père de Darius. Il faudra donc s'interroger sur ces ajouts. Auparavant, on notera que ce qui prévaut dans la démarche du compilateur est la volonté de brièveté. En effet, ce sont les chapitres les plus courts du Doop qui sont le plus fidèlement traduits. Dès que sa source fait de trop longues énumérations, fournit un ensemble de détails que Brunetto juge trop long ou redondant, il propose un résumé ou omet des parties entières du texte-source.

OMETTRE. .

On rencontre deux types de raccourcis dans le Tresor. Le premier porte sur une information partielle, une qualité, une précision, que Brunetto doit juger surabondante; dans le cas d'Esther par exemple, il omet de rappeler qu'elle est de la tribu de Benjamin, qu'elle fut ensevelie dans la cité des Mèdes :

\begin{tabular}{|c|c|}
\hline Doop (62) & Livre dou Tresor (58) \\
\hline $\begin{array}{l}\text { Hester, regina, filia fratris Mardochei, de } \\
\text { stirpe Benjamin captiva de Hierusalem in } \\
\text { urbe Susis translata atque ob aspectu formae } \\
\text { et praespicua uirginitatis excellentia regi } \\
\text { Persarum conubiis copulata; haec, ut } \\
\text { populum suum a periculo liberaret, morti se } \\
\text { obtulit amicumque regi aduersus populum } \\
\text { Dei nefaria persuadentem cruci tradendum } \\
\text { persuasit, gentemque liberam ab excidio } \\
\text { eruit, a seruitute eripuit. Iacet sepulta in } \\
\text { Susis urbe Medorum, in qua etiam et } \\
\text { regnauit. }\end{array}$ & $\begin{array}{l}\text { De Hester } \\
\text { Hester fu roine et fu fille dou frere } \\
\text { M[a]rdo[c]heus; et fu menee en prison de } \\
\text { Jerusalem en la cité de Sussi. Et por sa grant } \\
\text { biauté fu elle menee a [Assu]eres roi de } \\
\text { Perse. Ele se offri a mort por le peuple } \\
\text { sauver, et crucefia Amaim qui voloit destruire } \\
\text { le pueple Israel, et ensi le delivra de mort et } \\
\text { de servaige. Puis fu ele ensevelie en Sussi [ou } \\
\text { ele avoit regné]. }\end{array}$ \\
\hline
\end{tabular}

Il omet ainsi beaucoup de détails, qu'il juge sans doute non essentiels : lorsque le latin précise par exemple que le père d'Élie n'est pas Amos le prophète, 
d'origine modeste et de oppido Thecue, Brunetto ne mentionne pas ce détail. Il n'indique pas que Jean-Baptiste est de la tribu de Lévi, de Jérusalem, que Philippe, Pierre sont nés en la cité de Bethsaïde, que Jérémie fut lapidé près de «Tafné »", etc.

Il semble également que Brunetto veuille gommer certains éléments qui pourraient paraître redondants; il évite par exemple de rappeler, comme le fait le Doop, que Salomon est fils de David et de Bethsabée, dans le chapitre sur Salomon, car il l'a dit dans le précédent, portant sur David.

La seconde manière de Brunetto consiste à véritablement rayer un passage entier, comme, entre autres, dans le chapitre consacré à Daniel, ou celui dévolu à saint Paul, où le passage suivant du Doop n'est quasiment pas traduit par Brunetto :

[...] ex lupo ouis, inter apostolos uocatione nouissimus, praedicatione primus, in lege Gamalielis discipulus, in euangelio Christi seruus ; cuius patria Iudaea fuit, genus de tribu Beniamin. Hic secundo post ascensionem Domini anno baptizatus, dignitatem meruit apostolatus atque plus omnibus laborans multo latius inter ceteros Verbi gloriam seminauit atque doctrinam euangelicam sua praedicatione conpleuit. ${ }^{25}$

On peut citer également le passage suivant sur Jacques d'Alphée, omis par le Florentin :

[...] homo lucis, operarius ueritatis tantaeque etiam sanctitatis ut fimbriam uestimenti eius certatim cuperent adtingere populi. [...] quem Iosephus tantae sanctitatis in Iudaea perhibet extitisse, ut propter eius interfectionem Hierosolima credatur esse diruta. ${ }^{26}$

ou encore à propos de Timothée, pour qui Brunetto ne traduit que la deuxième partie du chapitre :

\begin{tabular}{|l|l|}
\hline \multicolumn{1}{|c|}{ Doop (84) } & \multicolumn{1}{|c|}{ Livre dou Tresor (83) } \\
\hline Timotheus, Ephesorum episcopus, de ciuitate & Tymoteu fu li secont deciples Pol, car il \\
Listrensium; patre Graeco, id est ethnico, & meesmes le mena de sa enfance avec soi et le \\
matre autem Iudaea, ut refert apostolus & batoia; et cil guarda virginité et chasteté. Nez \\
dicens: "Quae habitauit fides in auia tua & fu de la cité de [L]ist[e]nois, et fu ensevelis \\
Loide et matre tua Eunice, certum autem & en Epheson .x. jors a l'issue d'aoust \\
quod et in te. " Hic autem fuit discipulus & \\
Pauli eiusque spiritalis filius, quem puerum & \\
proprie secum idem Paulus adsumpsit; qui & \\
pudicus et uirgo permansit quique apud & \\
Ephesum in monte qui uocatur Sion cum & \\
magno honore sepultus quiescit.
\end{tabular}

\footnotetext{
${ }^{24} \mathrm{Il}$ semble ici qu'il y ait confusion entre le nom de la déesse égyptienne et un toponyme.

${ }^{25}$ Éd. cit., p. 199.

${ }^{26}$ Ibid., p. 213.
} 
Le procédé de coupe intervient également régulièrement lorsque le latin donne des listes, de qualités en général, que Brunetto écourte volontiers, comme dans le cas d'Élie :

\begin{tabular}{|l|l|}
\hline \multicolumn{1}{|c|}{ Doop (35) } & \multicolumn{1}{|c|}{ Livre dou Tresor (46) } \\
\hline Helias Thesbites, sacerdos magnus atque & Helyas Tesbitem fu granz prestres et prophete \\
propheta, habitator solitudinis, fide plenus, & que touzjors habita sous en desers, et plains \\
deuotione summus, in laboribus fortis, in & de foi et de sainte pensee. \\
industria sollers, excellenti ingenio praeditus, & \\
in exercitatione disciplinae strictus, in sancta & \\
meditatione adsiduus, metuque mortis & \\
intrepidus. & \\
\hline
\end{tabular}

Mais la rature est une méthode somme toute assez radicale; d'ailleurs, à y regarder de près, elle n'est jamais totale et, comme la plupart des compilateurs de son temps, Brunetto travaille avec plus de finesse : il condense par endroit, saute des informations, en précise d'autres, croise ses sources...

TRAVAILlER LE TEXTE...

Résumé

C'est régulièrement que Brunetto résume sa source, en particulier pour condenser des listes données par le latin. Ainsi, la suite de qualités prêtées à Salomon dans le Doop: uocatus sapientissimus omnium et dominici dedicator templi ; felix imperio, paternis mentis inpar, dilectus a Deo, sapiens in iudicio, in sententia iustus, pacificus in regno ${ }^{27}$, devient dans le Tresor " home tres glorious, plains de toutes sapiences, riches de tresor et de tres haute chevalerie $»^{28}$. À titre d'illustration, voici l'exemple de saint Paul :

\begin{tabular}{|l|l|}
\hline \multicolumn{1}{|c|}{ Doop (68) } & \multicolumn{1}{|c|}{ Livre dou Tresor (72) } \\
\hline $\begin{array}{l}\text { Ob amorem quoque Christi multas passiones } \\
\text { grauiaque sustenuit corporis tormenta: in }\end{array}$ & Il sofri por le nom de Dieu fain, soif, nuese, et \\
primis iudaicas persecutiones et gentium & nuit; il soffri la rage des bestes sauvaiges et \\
miserias, et laborem, famem, sitim, frigus et & maintes fe[ru]es et tormenz de chartres. Les \\
nuditatem, diei noctisque profundum, & juys le trairent et fu lapidez a mort; il fu \\
naufragia et mille pericula, rabiem ferarum, & enchaenez en une prison, dont il fu desliez et \\
uerbera, carcerales tenebras et tormenta & calé par le mur avec un cofin. A la fin le fist \\
catenarum, squalores et uincula. Inter haec & l'empereur Noiron decoler le jor que saint \\
ligatur a Iudaeis, traditur gentibus, lapidatur & Pieres fu crucefiez. \\
ad necem, in sportam per murum dimittitur, & \\
uirgis caeditur, poenis artatur, in carcerem & \\
uinctus terraemotu facto resoluitur. Ad & \\
ultimum a Nerone gladio caeditur eo die quo & \\
et Petrus crucifixus est & \\
\hline
\end{tabular}

${ }^{27}$ Éd. cit., p. 155.

${ }^{28}$ Éd. cit., p. 80. 
Précisions, compléments

Il est toujours délicat, lorsque l'on veut caractériser le travail des compilateurs médiévaux, de définir un système global. En effet, les auteurs se réclament souvent d'une certaine brièveté ; celle-ci, comme je l'ai montré ailleurs, n'est pas nécessairement en rapport avec notre concept de 'faire bref' et renvoie à l'écriture encyclopédique elle-même ${ }^{29}$. Cependant, la déclaration de brièveté correspond aussi à un réel souci de condensation de la matière qui apparaît clairement dans la démarche de résumé, de coupe mise en œuvre par rapport aux sources. Mais, en parallèle de cette écriture du raccourci, existe la volonté de préciser la source, d'ajouter quelque détail, en utilisant pour ce faire d'autres sources ou, plus simplement, la mémoire du compilateur. Brunetto Latini n'échappe pas à cette règle et l'applique avec sa translation du Doop. On peut classer ses ajouts, grosso modo, en trois catégories: 1) un apport visant à préciser une donnée particulière ou un ensemble d'éléments ; 2) une précision calendaire de la mort du personnage ; 3) une explication étymologisante du nom.

- Le premier cas est parfaitement traditionnel dans toute écriture encyclopédique médiévale; on peut considérer dans cette catégorie deux sousensembles. Le premier concerne des 'corrections de détail'. Les informations sont éparpillées au fil du texte, portant sur le nom, le lieu, une action particulière, etc. Je me contenterai donc de donner quelques exemples, qu'il est possible de multiplier, ces exemples parlant d'eux-mêmes.

\begin{tabular}{|c|c|}
\hline Doop & Livre dou Tresor \\
\hline $\begin{array}{l}\text { Saint Jean-Baptiste } \\
\text { Iohannes Baptista, filius Zachariae [...] qui } \\
\text { necdum editus Christum prophetauit, } \\
\text { salutauit ex utero, in columba agnouit, in } \\
\text { deserto monstrauit. }\end{array}$ & $\begin{array}{l}\text { Helysabeth cosine Marie engenera de } \\
\text { Zacharie son mari un fiz qui ot a nom Johan } \\
\text { [...] il prophetiza Dieu ainz qu'il nasquist et } \\
\text { le salua dedenz le ventre sa mere; il conut } \\
\text { Jesu a la colomb[el et le mostra au doi. Il } \\
\text { meesmes le batoia et por ce est [il] apelez } \\
\text { Johan Baptiste. }\end{array}$ \\
\hline $\begin{array}{l}\text { Judith } \\
\text { haec pro salute populi morti se obtulit nec } \\
\text { trepidauit furorem regium, nam dormientem } \\
\text { necauit principem }\end{array}$ & $\begin{array}{l}\text { Ele ne douta pas le furor des rois, ainz se offri } \\
\text { a la mort por sauver le pueple, car ele occist } \\
\text { Olofernem quant il dormoit. }\end{array}$ \\
\hline $\begin{array}{l}\text { Esther } \\
\text { [...] et praespicua uirginitatis excellentia regi } \\
\text { Persarum conubiis copulata. }\end{array}$ & $\begin{array}{l}\text { Et por sa grant biauté fu elle menee a } \\
{[\text { Assu]eres roi de Perse }}\end{array}$ \\
\hline
\end{tabular}

29 B. Ribémont, Les Origines des encyclopédies médiévales. D’Isidore de Séville aux Carolingiens, op. cit.; "Around the definition of an encyclopedic genre in the Middle Ages », art. cit.. 
Le deuxième sous-ensemble correspond à une pratique de croisement des sources et implique un emboîtement de données. La translation du Doop est alors juxtaposée à un autre morceau de texte ou imbriquée dans celui-ci.

Dans le chapitre consacré à Judith, Brunetto traduit quasiment mot à mot Doop 63 ; il rajoute le célèbre épisode d'Holopherne et de la tête tranchée, d'après le Livre de Judith. Les chapitres consacrés à la Vierge sont le fruit d'un assemblage d'informations tirées de la Bible, des Étymologies d'Isidore, de la Légende dorée ou de sources communes à Brunetto et à Jacques de Voragine, et de Doop 66. Le chapitre 64 du Tresor «Dou parenté Nostre Dame» contient les mêmes données que la Légende dorée $e^{30}$. En particulier, l'identification de la sépulture de Marie ne vient pas du Doop, qui au contraire dit que le lieu de la tombe de Marie n'est pas connu (Quod idem incertum est [...] specialiter tamen nulla docet historia Mariam gladii animaduersione peremptam, quia nec obitus eius uspiam legitur, dum tamen repperiatur sepulcrum). La vallée de Josaphat est l'endroit qui apparaît dans un oracle de Joël, où Dieu rassemblera les nations le jour du Jugement dernier (Joël, 4, 2 et 12). Brunetto Latini tire cette information, soit directement de Jérôme, soit, plus probablement de la Légende dorée ${ }^{31}$ ou de sources communes, Papias ou Huguccio de Pise par exemple. Dans son chapitre 65, «La premiere Marie mere de Dieu », il rappelle l'étymologie stella maris, qu'il tient d'Isidore (Étymologies 7, 10, 1). Dans le chapitre sur Jean-Baptiste (66), Brunetto ne reprend pas directement le Doop pour l'épisode de Salomé ; il mélange un peu les noms, confondant la fille et l'épouse. La présentation de la tête à Hérodias par sa fille vient de l'Évangile (Matt. 14, 11 ; Marc 6,28 ) ou bien Brunetto continue d'exploiter Jacques de Voragine ${ }^{32}$. Dans le chapitre sur saint Pierre, Brunetto ajoute des informations tirées des Étymologies: «Piere vaut autant a dire come conoissan[s], por ce que il conut Dieu quant il dist: 'tu es Crist filz de Dieu vivant' $\|^{33}$; quia dixerat Petrus : 'Tu es Christus filius Dei vivi ${ }^{34}$. On peut encore multiplier les exemples de ce travail de compilation croisée, dans lequel, on le remarquera, les Étymologies occupent aussi une bonne place.

Dans ce jeu croisé avec les sources, il arrive parfois même, que le Doop n'apparaisse qu'à l'état de trace - cas rare et le plus extrême - comme dans le chapitre sur David:

${ }^{30}$ Le texte de Jacques a été composé entre 1261 et 1266 ; il est tout à fait possible que Brunetto l'ait connu. À moins qu'il y ait des sources communes. Si l'on compare le texte de Brunetto avec le chapitre de Jacques de Voragine sur la nativité de la bienheureuse Vierge Marie, on peut mesurer les points de concordance (voir par exemple la traduction J.-B. M. Rozé, t. 2, Paris, GF, 1967, p. 172-3). Dans son chapitre 63, Brunetto note que Joseph n'intervient pas dans la conception du Christ et sur la coutume des Hébreux; on trouve la même chose au début du chapitre sur la nativité de la bienheureuse Vierge Marie de la Légende dorée (trad. cit., p. 171). La mère de la Vierge (Tresor 64) n'apparaît pas dans le Nouveau Testament. Jacques de Voragine reprend une information venant des Évangiles apocryphes, comme le Protévangile de Jacques ( $\mathrm{II}^{\mathrm{e}} \mathrm{s}$.) et le Pseudo-Matthieu (VI ${ }^{\mathrm{e}} \mathrm{s}$.). Brunetto tient donc son information de la source des apocryphes ou de La Légende dorée.

${ }^{31}$ « Assomption de la bienheureuse Vierge Marie », trad. cit., p. 91.

${ }^{32}$ Trad. cit., p. 154.

${ }^{33}$ Éd. cit., p. 102.

${ }^{34}$ Étym., 7, 9, 2. 


\begin{tabular}{|c|c|}
\hline Doop (33) & Livre dou Tresor (44) \\
\hline $\begin{array}{l}\text { Dauid, idem rex et propheta, ortus de genere } \\
\text { Iuda, filius Iesse, natus in Bethleem, puer } \\
\text { pastorius, inter fratres aetate iunior, mente } \\
\text { praestantior, a Domino in regno uocatus, a } \\
\text { propheta in rege unctus, belliger iunenis; in } \\
\text { certamine singularis, in triumpho gloriosus, } \\
\text { uincendo ueteranus, patiens in aduersis, } \\
\text { prudens in periculis, in peccato proprio } \\
\text { dolens, in alieno funere lugens, pronus ad } \\
\text { poenitentiam, uelox ad ueniam, in conuicio } \\
\text { mitis, ad misericordiam facilis. Qui inimicum } \\
\text { regem, dum posset, non nocuit, sed tantum } \\
\text { reseruauit traditum et uindicauit occisum. } \\
\text { Hic leonem et ursum sine gladio interfecit, } \\
\text { citharae suauitate inmundum spiritum } \\
\text { depulit, gigantes expugnauit. Igitur } \\
\text { quadragensimo interea regni ac } \\
\text { septuagensimo aetatis anno diem uitae suae } \\
\text { supremum consummauit, sepultusque est in } \\
\text { ciuitate sua Bethleem, quae alio uocabulo } \\
\text { dicitur Efrata, ubi etiam Dominus noster } \\
\text { Iesus Christus secundum carnem est genitus, } \\
\text { ibi etiam et Rachel, quum Beniamin } \\
\text { peperisset, occubuit, ibi etiam et sepulcrum } \\
\text { Iesse patris Dauid ostenditur. Est autem hic } \\
\text { locus in tribu Iuda, sexto ab Hierusalem } \\
\text { miliario positus contra meridie, in itinere quo } \\
\text { pergitur Hebron. }\end{array}$ & $\begin{array}{l}\text { David fiz Yesse, qui fu estrait de la lignee } \\
\text { Judas, nasqui en Bethleem, [et] occist } \\
\text { Goliam le geant qui enemis estoit au roi Saul, } \\
\text { qui fu sires de Jerusalem et de toz les juys. Il } \\
\text { vanqui sens coutel le lion et l'orse et vanqui } \\
\text { le jaant, et maintes autres granz choses fist } \\
\text { il ; por quoi Saul le heoit et le chaçoit por } \\
\text { [lui] tolir la vie, car il doutoit que il ne le } \\
\text { tosist son regne. Mes si come a Dieu plot, } \\
\text { Saul morut et David fu rois aprés lui et fu } \\
\text { molt victorious. Et Dieu vost que il fust rois } \\
\text { et prophete. Et ja fust il pecheor, il revenoit } \\
\text { en pénitence tost et volentiers. Il aima } \\
\text { Bersabee la feme Urie son conostable; et [en } \\
\text { la] fin fist [il] aler Urie en une bataille ou il } \\
\text { morut, et puis tint la feme et en lui engendra } \\
\text { il Salemon le saige, qui fu rois aprés lui. Et } \\
\text { sachiez que David fu li soverains prophetes } \\
\text { de touz, car il ne prophetiza pas a la maniere } \\
\text { des autres. Car prophetie est en .iiii. } \\
\text { manieres : ou en fait ou en diz, ou en vision, } \\
\text { ou en songe. En faiz fu l'arche que Noe fist, } \\
\text { qui fu senefiance de sainte Yglise ; en diz fu } \\
\text { ce que li angles dist a Abraam, en sa semence } \\
\text { seront toutes genz beneoites; en vision fu le } \\
\text { rube que Moyses vit ardoir; en songe furent } \\
\text { les .vii. vaches et les .vii. spies que Pharaon } \\
\text { sonja, sor quoi Joseph prophetiza. Mes hors } \\
\text { de ces .iiii. manieres prophetiza David par } \\
\text { soule interpretacion de Dieu et dou Saint } \\
\text { Esperit, qui le enseingna a dire tote la } \\
\text { naissance [Jesu Crist et sa mort et sa } \\
\text { resurrection]. Il descovri ce que les autres } \\
\text { avoient dit covertement, selonc [ce] que l'en } \\
\text { puet veoir en son livre, qui est apelez Sautier, } \\
\text { en semblance d'un estrument qui autresi a } \\
\text { nom sautier, qui a .x. cordes : autresi parole li } \\
\text { livres de .x. comandemenz en.cl. saumes qui } \\
\text { sont au Sautier. Et sachiez que David regna } \\
\text {.xl. anz et passa de cest siecle en l'aage de } \\
\text { [.lx.] anz. }\end{array}$ \\
\hline
\end{tabular}

- Les chapitres du Tresor présentent régulièrement, en sus de la version commune du Doop, des informations traditionnelles des martyrologes sur les dates de décès et les lieux de sépulture des personnages mentionnés. Pour les personnages 
de l'Ancien Testament, seul le lieu de sépulture est mentionné; c'est le cas de David, Salomon, Élisée, Isaïe, Ezéchiel, Achias, Jado, Tobie, les Trois enfants, Esdras, Zorobabel, Esther, Judith, Zacharie. Si seuls les lieux d'ensevelissement de Jean-Baptiste et d'André sont donnés, si Brunetto ne donne pas de précision quant à Tite, apparaissent les dates de fêtes pour toutes les autres figures du Nouveau Testament. Certaines de ces informations se trouvent dans le Doop lui-même, et c'est le cas de presque tous les personnages de l'Ancien Testament: Salomon (sepultusque est in ciuitate patris sui Bethleem), Élisée (cuius sepulcrum usque hodie in Sebastia ciuitate ostenditur), Isaïe (Iacet sub quercu Rogel), Ezéchiel (Sepultus autem est a populo in agro Maur in sepulcro Sem et Arfaxat), Jado (sepultumque tenet Bethel), Tobie (Iacet in Nineue sepultus in pace), Trois enfants (Iacent in Babylonia pariter sub uno specu positi et magna cum ueneratione sepulti), Esdras (Iacent in Babylonia pariter sub uno specu positi et magna cum ueneratione sepulti), Zorobabel (idem et ipsi in Hierusalem sepulti quiescunt), Esther (Iacet sepulta in Susis urbe Medorum), Judith (Vixit annis centum quinque sepultaque est in spelunca uiri sui Manasse in Bethulia ciuitate), Zacharie et Élisabeth (requiescunt in Hierusalem). Brunetto reprend également le Doop pour Jean-Baptiste (Sepultus est in Sebastia).

Pour les autres figures du Nouveau Testament, les choses diffèrent ; Brunetto peut emprunter certaines informations à la version commune du Doop concernant surtout le lieu de sépulture, puis il y rajoute ou précise des dates; mais parfois, de façon qui peut étonner, il omet un lieu, tout en donnant une date qu'il adjoint (exemple de Jacques, fils de Zébédée) :

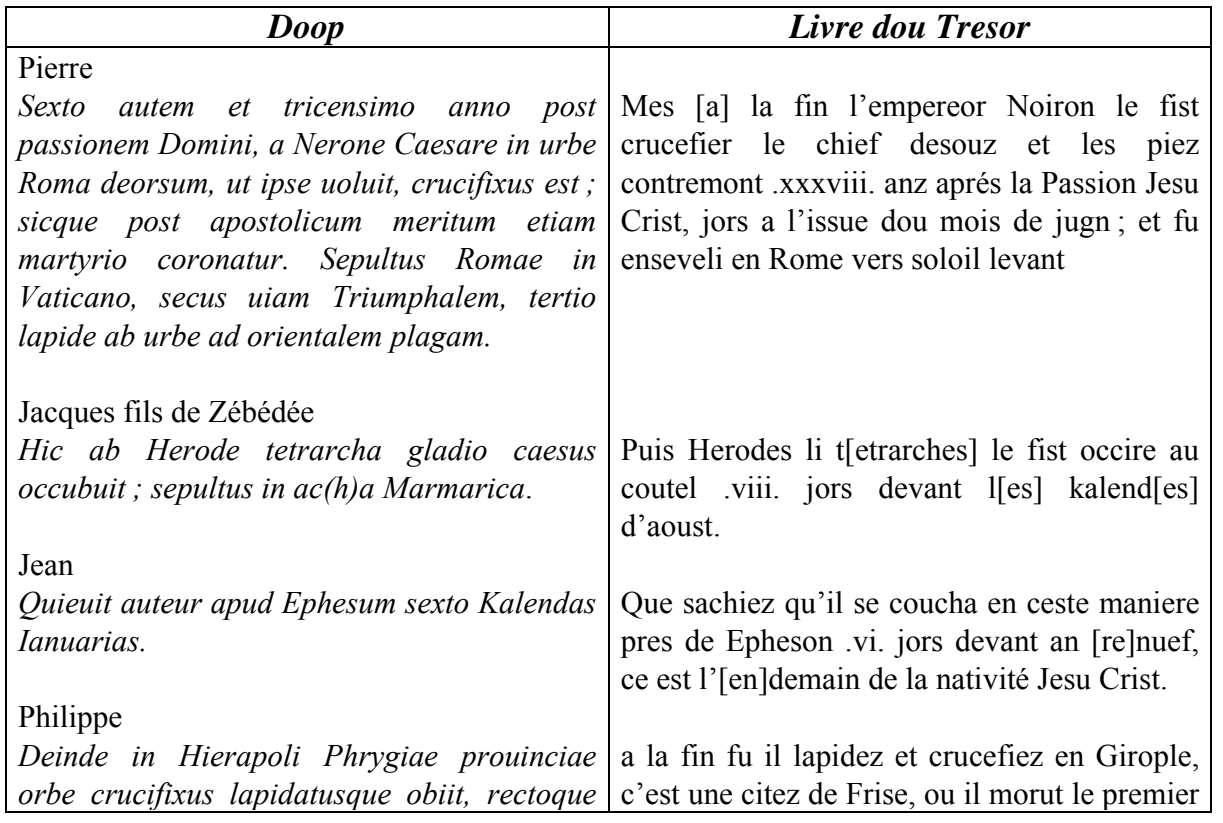

${ }^{35}$ Voir éd. cit., p. 106, n. 7. 


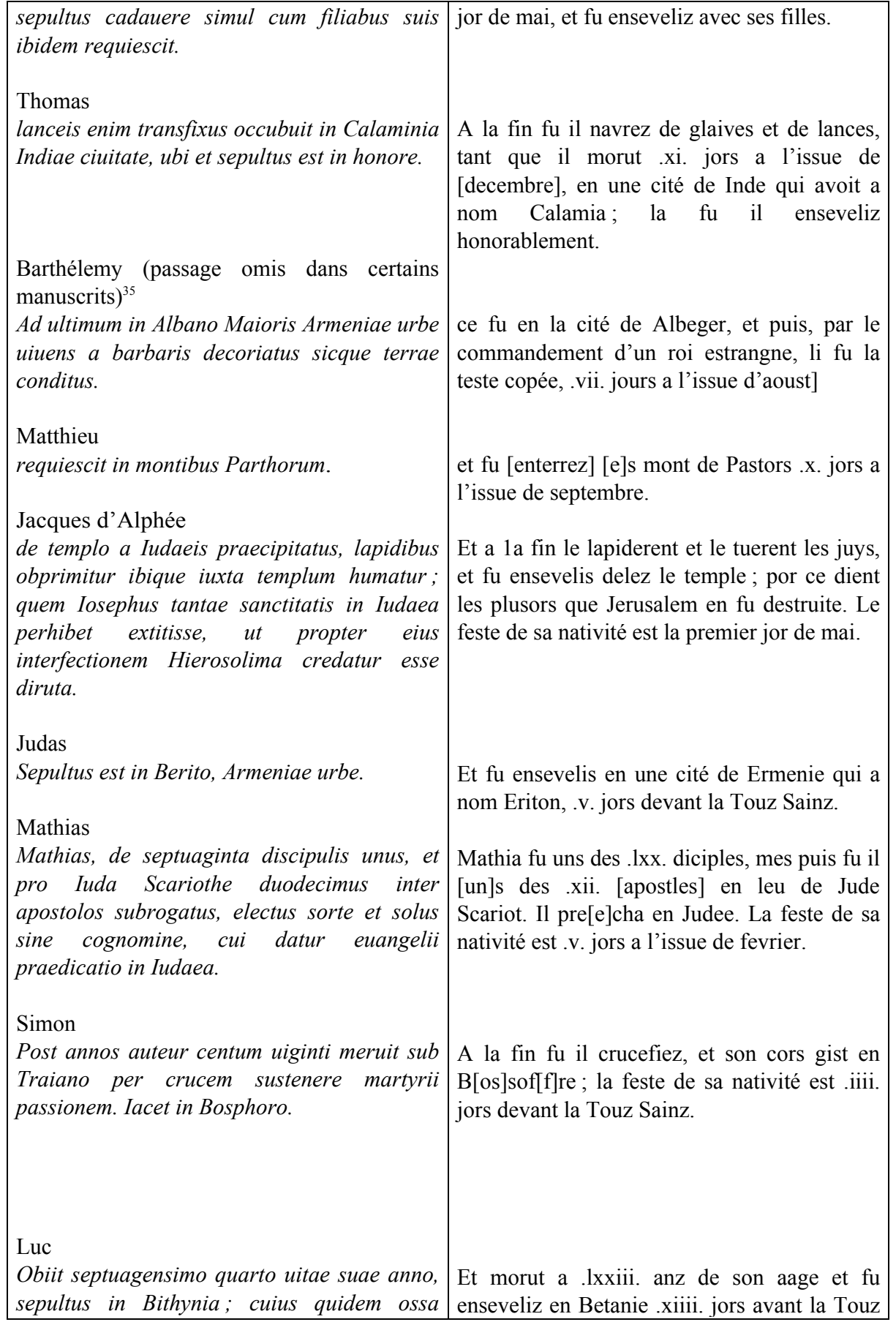




\begin{tabular}{|l|l|}
\hline $\begin{array}{l}\text { regnante Constantio in Constantinopoli sunt } \\
\text { translata. }\end{array}$ & $\begin{array}{l}\text { Sainz; mes ses os en furent portez en } \\
\text { Costantinople au tens de l'empereor } \\
\text { Constantin. }\end{array}$ \\
$\begin{array}{l}\text { Marc } \\
\text { quiete sepultus. }\end{array}$ & $\begin{array}{l}\text { Il fonda premierement] Iglise en Egypte et } \\
\text { morut au tens Noiron, .vi. jors a l'issue } \\
\text { d'avril. }\end{array}$ \\
$\begin{array}{l}\text { La feste de sa nativité est .xii. jors [a] l'entree } \\
\text { de jugn. }\end{array}$ & $\begin{array}{l}\text { Nez fu de la cité de [L]ist[e]nois, et fu } \\
\text { apud Ephesum in monte qui uocatur Sion cum } \\
\text { magno honore sepultus quiescit. } \\
\text { d'aoust. en Epheson .x. jors a l'issue }\end{array}$ \\
$\begin{array}{l}\text { Tite } \\
\text { quem ad instruendas Cretae ecclesias } \\
\text { praefatus reliquit apostolus, ibique in Pace } \\
\text { defunctus est atque sepultus. }\end{array}$ & $\begin{array}{l}\text { et il le laissa por destruire les ydoles de Grece } \\
\text { enser edifier yglises. Et la morut il et fu } \\
\text { enseliz en pes. }\end{array}$ \\
\hline
\end{tabular}

- Nous avons pu constater plus haut, à propos de Zacharie, que Brunetto inscrivait une étymologie qui ne figure pas dans la version commune du Doop. Il est effectif que le Florentin fournit de nombreuses étymologies pour les noms qu'il cite, tout particulièrement pour les personnages du Nouveau Testament, la plupart ne figurant pas dans la version commune du Doop. Une telle pratique cependant rapproche évidemment le discours de Brunetto de la méthode isidorienne et illustre en soi l'influence de l'évêque de Séville. On pourrait seulement être surpris que le Doop ne propose quasiment pas d'étymologies ${ }^{36}$. Il y en a cependant quelques-unes, évidemment traduites et/ou adaptées par Brunetto (sauf pour Barthélemy, dont l' 'étymologie' reste vague) :

\begin{tabular}{|l|l|}
\hline $\begin{array}{l}\text { André } \\
\text { Andreas, qui interpretatur decorus }\end{array}$ & $\begin{array}{l}\text { Andreas vaut tant a dire en grezois come } \\
\text { biaus. }\end{array}$ \\
$\begin{array}{l}\text { Marie } \\
\text { Maria, quae interpretatur domina siue } \\
\text { inluminatrix }\end{array}$ & $\begin{array}{l}{[\ldots] \text { son nom vaut tant a dire come [...] dame }} \\
\text { et clarté. }\end{array}$ \\
$\begin{array}{l}\text { Thomas } \\
\begin{array}{l}\text { Thomas, Christi didymus nominatus et iuxta } \\
\text { latinam linguam Christi geminus ac similis } \\
\text { saluatori audiendo incredulus, uidendo } \\
\text { fidelis. }\end{array}\end{array}$ & $\begin{array}{l}\text { Thomas vaut tant a dire come abysme, et il ot } \\
\text { en sornom Didimus, qui vaut autant a dire } \\
\text { come doutans, car il se douta de la resurection } \\
\text { Jesu Crist jusques a tant qu'il bota ses mains } \\
\text { dedenz les plaies. }\end{array}$ \\
\hline
\end{tabular}

\footnotetext{
${ }^{36}$ Celle d'André, présente dans le Doop, est aussi dans les Étymologies(7, 9, 11), mais renvoyant à l'hébreu : Secundum Hebraeam etymologiam interpretetur decorus.
} 


\begin{tabular}{|l|l|}
\hline $\begin{array}{l}\text { Barthélemy } \\
\text { Bartholomeus, apostolus, nomen ex Syra } \\
\text { lingua suscipiens. }\end{array}$ & \\
$\begin{array}{l}\text { Jacques } \\
\text { Iacobus Alfei, episcopus Hierosolimorum } \\
\text { primus, cognomento Iustus, sororis matris } \\
\text { Domini filius, unde et Domini frater uocatus. }\end{array}$ & $\begin{array}{l}\text { Jaques Alphey fu fiz de la s[e]conde Marie, } \\
\text { seror de la mere Dieu, et por ce est il apelés } \\
\text { frere Damedieu. Et son nom vaut tant a dire } \\
\text { come justes, et ensi ot il en sornom aucune } \\
\text { foiz. }\end{array}$ \\
\hline
\end{tabular}

À côté de ces adaptations plus ou moins directes, Brunetto reprend des informations biographiques fournies par le Doop qu'il 'étymologise' véritablement ; c'est le cas de Simon et de Luc :

\begin{tabular}{|l|l|}
\hline $\begin{array}{l}\text { Simon } \\
\text { Simon Zelotes, qui prius dictus est } \\
\text { Chananaeus }\end{array}$ & $\begin{array}{l}\text { Symon Zelotes vaut autant a dire come } \\
\text { cananeus ou possession. }\end{array}$ \\
$\begin{array}{l}\text { Luc } \\
\text { Lucas, euangelista et apostolicae conscriptor } \\
\text { historiae; natione Syrus, arte medicus }\end{array}$ & $\begin{array}{l}\text { Lucas evangeliste vaut autant a dire come } \\
\text { mires }\end{array}$ \\
\hline
\end{tabular}

Mais, le plus fréquemment, le Livre dou Tresor fournit des étymologies ne figurant pas dans la version commune du Doop ${ }^{37}$. La première question qui se pose par rapport à ces ajouts est de savoir s'ils sont authentiquement isidoriens et si on peut les retrouver dans les Étymologies. C'est effectivement le cas pour certaines, à quelques confusions près : par exemple, Brunetto (ou son manuscrit source) confond Helisaeus et Hysaias: on trouve en effet en Étymologies, 7, 8, 6: Helisaeus Domini

${ }^{37}$ En voici la liste :

- Helyseus vaut tant a dire come filz de mon Dieu.

- Hysaias vaut tant a dire come salut dou Seignor

- Ezechiel vaut autant a dire come force de Dieu

- Daniel le prophete vaut autant a dire come jugement de Dieu ou home amable

- Thobias prophete vaut autant a dire come bien de Dieu.

- E[sd]ras vaut autant a dire come [e]defiemen[z] de Jerusalem

- Zacharias vaut autant a dire come memoire de Damedieu

- Machabeu vaut tant a dire come noble [et] trionphanz.

- Pieres ot .ii. nons, car il ot a nom Symon Pieres. Et Symon vaut autant a dire come obeissant, por ce que il obei a Dieu lors que il dist : "vien aprés moi ». Piere vaut autant a dire come conoissan[s], por ce que il conut Dieu quant il dist : « tu es Crist filz de Dieu vivant ».

- Pol, son nom vaut tant a dire come mervoillous

- Joham Evangeliste fu fiz Zebedieu de la tierce Marie, et frere Jaque ; et son nom vaut tant a dire come grace de Dieu.

- Phelipe vaut tant a dire come bo[uc]he de lampe.

- Lucas evangeliste vaut autant a dire come [...] luissanz

- Marcus Evangeliste vaut autant a dire come granz.

- Barnabe avoir a nom premierement Joseph, et vaut autant a dire come feel. 
salus interpretatur / Tresor "Hysaias vaut tant a dire come salut dou Seignor». Mais on a : Ezechiel fortitudo Dei (Étym., 7, 8, 9) / Tresor: «Ezechiel vaut autant a dire come force de Dieu »; Zacharias memoria Dei (Étym., 7, 8, 20) / Tresor: «Zacharias vaut autant a dire come memoire de Damedieu»; Philippus os lampadarum, vel os manuum (Étym., 7, 9, 16) / Tresor: «Phelipe vaut tant a dire come bo[uc]he de lampe ».

Il y a donc incontestablement une influence des Étymologies d'Isidore. Mais est-elle directe ? On a vu en effet qu'il pouvait y avoir des points de contacts entre le Tresor et la Légende dorée. Or ce dernier texte propose aussi des étymologies qui sont très proches de celles de l'ouvrage de Brunetto. L'étymologie de Barnabé par exemple, identifié à filius dans la Légende dorée, donne «feel » chez Brunetto suite à une mauvaise lecture.

Il reste aussi à s'interroger, toujours dans le cadre de la réception d'Isidore qui nous préoccupe ici, sur la tradition pseudo-isidorienne du Doop ${ }^{38}$. Dans l'édition d'Arévalo reproduite par Migne ${ }^{39}$, figure une annexe au Doop, qui contient un certain nombre de rubriques établies d'après des éditions modernes qui, très certainement, intègrent des interpolations d'ordre étymologique. Il est alors intéressant de comparer les affirmations de Brunetto avec ces dernières.

\begin{tabular}{|c|c|}
\hline Helyseus vaut tant a dire come filz de mon & Eliseus, qui interpretatur Domini mei salus \\
\hline $\begin{array}{l}\text { Dieu. } \\
\text { Hysaias vaut tant a dire come salut dou } \\
\text { Seignor }\end{array}$ & Isaias, [...] Domini salus interpretatur \\
\hline $\begin{array}{l}\text { Ezechiel vaut autant a dire come force de } \\
\text { Dieu }\end{array}$ & Ezechiel fortitudo Dei interpretatur \\
\hline $\begin{array}{l}\text { Daniel le prophete vaut autant a dire come } \\
\text { jugement de Dieu ou home amable }\end{array}$ & Daniel, filius Abda, judicium. \\
\hline $\begin{array}{l}\text { Thobias prophete vaut autant a dire come } \\
\text { bien de Dieu. }\end{array}$ & Tobias propheta sanctus, Dei summi servus \\
\hline $\begin{array}{l}\text { Zacharias vaut autant a dire come memoire de } \\
\text { Damedieu }\end{array}$ & $\begin{array}{l}\text { Zacharias propheta, qui interpretatur memor } \\
\text { Domini }\end{array}$ \\
\hline $\begin{array}{l}\text { Pieres ot .ii. nons, car il ot a nom Symon } \\
\text { Pieres. Et Symon vaut autant a dire come } \\
\text { obeissant, por ce que il obei a Dieu lors que il } \\
\text { dist: « vien aprés moi ». Piere vaut autant a } \\
\text { dire come conoissan[s], por ce que il conut } \\
\text { Dieu quant il dist: «tu es Crist filz de Dieu } \\
\text { vivant». }\end{array}$ & $\begin{array}{l}\text { Simon Petrus tribus nominibus appellatur. } \\
\text { Primo a parentibus Cephas, deinde Simon, } \\
\text { tertio Petrus. His autem duobus nominibus } \\
\text { nominavit illum Deus: Simon, qui } \\
\text { interpretatur obediens; Petrus, agnoscens. }\end{array}$ \\
\hline $\begin{array}{l}\text { Pol, son nom vaut tant a dire come } \\
\text { mervoillous }\end{array}$ & Paulus, quod interpretatur mirabilis \\
\hline $\begin{array}{l}\text { Joham Evangeliste fu fiz Zebedieu de la } \\
\text { tierce Marie, et frere Jaque ; et son nom vaut } \\
\text { tant a dire come grace de Dieu. }\end{array}$ & $\begin{array}{l}\text { Joannes apostolus, et evangelista, qui } \\
\text { interpretatur gratia Dei, }\end{array}$ \\
\hline
\end{tabular}

\footnotetext{
${ }^{38}$ Je n'ai malheureusement pas pu consulter le ms. G.

${ }^{39}$ P.L. 83, c. $1275-1293$.
} 


\begin{tabular}{|c|c|}
\hline $\begin{array}{l}\text { Phelipe vaut tant a dire come bo[uc]he de } \\
\text { lampe. } \\
\text { Lucas evangeliste vaut autant a dire come } \\
\text { [...] luissanz } \\
\text { Marcus Evangeliste vaut autant a dire come } \\
\text { granz. }\end{array}$ & $\begin{array}{l}\text { Philippus, qui interpretatur os lampadis, } \\
\text { Lucas, qui interpretatur elevans, sive } \\
\text { consurgens, } \\
\text { Marcus, qui interpretatur excelsus }\end{array}$ \\
\hline
\end{tabular}

On voit donc que l'on retrouve certaines étymologies venant du livre des Étymologies d'Isidore (Philippe, Zacharias, etc.), d'autres qui ne sont pas tirées de cet ouvrage et que Brunetto reprend, d'autres enfin non utilisées par Brunetto, luimême en proposant qui ne figurent pas dans cette tradition. On remarquera que, par rapport aux Etymologies, apparaît la même confusion chez Brunetto et dans cette version du Doop à propos d'Isaïe.

\section{Conclusion}

Il est délicat, dans le cadre limité de cette enquête, de préciser davantage quant aux sources du Tresor; là n'est au demeurant pas l'objet de cet article. On peut affirmer en revanche que Brunetto a travaillé sur une forte base isidorienne pour écrire ses chapitres concernant l'histoire sainte. Il a compilé un ou, plus probablement, plusieurs manuscrits du Doop, avec une version longue et une version apparentée au manuscrit de Vérone, contenant le chapitre sur les Macchabées. Il peut avoir utilisé une version interpolée 'pseudo-isidorienne', incluant des étymologies et quelques données. Il peut avoir croisé sa source principale avec des chapitres de la Légende dorée ou - la chronologie étant très serrée ici - des textes utilisés par Jacques de Voragine, comme les œuvres encyclopédiques de Papias et de Huguccio de Pise. Mais le 'pseudo-isidorisme', comme la tradition des textes authentiques de l'évêque de Séville qui s'imposent en cette deuxième moitié de $\mathrm{XIII}^{\mathrm{e}}$ siècle, sont la marque du caractère particulièrement vivant de l'influence d'Isidore. L'exemple du Livre dou Tresor, une des toutes premières encyclopédies écrites directement en français, montre combien, dans l'univers encyclopédique, Isidore demeure une source, une référence et une autorité. La réception de celui qui est, avec ses Étymologies et son De natura rerum, le 'père fondateur' du genre encyclopédique médiéval, dépasse donc ces deux textes et c'est sans doute l'ensemble de l'œuvre de l'évêque de Séville qu'il faut prendre en considération dans l'étude d'une réception tardive beaucoup plus riche que l'on aurait pu le penser.

Bernard Ribémont

Université d'Orléans 